\title{
STRUCTURAL AND FUNCTIONAL INTERPLAY IN ANXIETY RELATED CLASSIFICATION: A GRAPH SIGNAL PROCESSING APPROACH
}

\author{
Giovanna Orrù, Pierre Maurel, Julie Coloigner
}

Univ Rennes, Inria, CNRS, Inserm

\begin{abstract}
Anxiety disorders are one of the most common mental health conditions with a high rate of everyday life disability. Connectivity is steadily gaining relevance to increase our knowledge of psychiatric diseases. Graph signal processing (GSP) is a new framework to integrate structural connectivity and brain function. We propose here a graph-based analysis using GSP metrics and classification procedure, to identify anxiety biomarkers. Results suggest that the joint consideration of structure-function features improves their discriminatory accuracy, and our understanding of the pathophysiology of anxiety.
\end{abstract}

Index Terms - functional and structural connectivity, neuropsychological biomarkers, graph signal processing

\section{INTRODUCTION}

Anxiety is a psychological condition that affects about 15 millions adults and can have a strong impact on their lives. Structural and functional characteristics of such psychiatric disorders have been studied using neuroimaging. In pediatric patients, changes in cortical thickness have been found in the prefrontal cortex, hippocampus, and amygdala, regions involved in emotional processing [1]. Anxiety can, indeed, be explained as an abnormal tuning of attention and emotional regulation, since it involves the activation of specific known networks. Advanced neuroimaging can provide increased sensitivity to measure such deficits and identify structural and functional neural pathways that are affected by anxiety.

Brain connectivity refers to the anatomical and functional links between brain regions. Diffusion-weighted imaging (DWI) enables the estimation of structural connectivity across brain regions using tractography algorithms. Moreover, functional connectivity is a widely used measure that characterizes functional interactions between brain regions, estimated using functional neuroimaging. Graph analysis provides a powerful way of quantifying neural pathways and evaluating functional brain networks. It consists in modeling the brain as a network where nodes are associated with regions of interest and edge strengths represent the degree of connectivity between pairs of regions [2]. Network-based algorithms provide parameters that characterize the global organization of the brain and its potential alterations. Formalizing the structural and functional organization enables the identification of distinctive patterns or modified reorganizations possibly linked to different diseases such as depression or anxiety [3]. Studies in the literature have traditionally analyzed functional and structural connectivity independently, however in recent studies, models integrating both structural and functional organization provided more specific and complementary characteristics [4].

A promising framework for multimodal analysis is Graph Signal Processing (GSP), in which classical signal processing (convolution, Fourier transform, filtering), can be applied to graphs, overcoming the obstacle of their irregular domain. Rather than focusing only on the structure of the graph, the GSP therefore also takes into account the functional signal defined on the underlying structure of the graph. From this perspective, GSP could be applied to take into consideration the joint structure-function nature of neuroimaging and help uncover hidden interplay that might link these two representation modalities. In recent years, this approach has been notably used to investigate how the structural substrate might constrain functional cognitive dynamics while incorporating realistic surrogate data [5]. In a similar way, GSP filtering was employed to describe the coupling with structure of functional signal decomposed on low and high variation of "graph eigenmodes" [6]. Other works have explored the use of GSP to discriminate neurological disorders [7, 8]. These studies using GSP metrics offer new directions and unprecedented insights for applications in clinical research and medical diagnosis.

In this paper, we provide a new approach based on GSP metrics exploiting simultaneously functional and structural connectivity. A classification pipeline based on support vector machine was proposed to identify biomarkers that could distinguish subjects affected by anxiety from controls.

\section{METHODS}

\subsection{Data}

The data used in this study was collected from the Human Brain Network dataset, available for pediatric mental health 
research [9]. Our dataset consists of 43 subjects, whose age spanned from 10 to 20 years, including 20 subjects suffering from anxiety and 23 control subjects. In this study, we analyzed the resting state functional magnetic resonance imaging (rs-fMRI) and DWI data.

\subsection{Preprocessing}

Functional and diffusion imaging scans were preprocessed using two open source medical image processing softwares: Anim: $\rrbracket^{1}$ and FMRIB Software Library (FSL). Standard preprocessing steps were applied: functional images were (1) slice-time corrected, (2) realigned to remove physiological motion, (3) intensity normalized and (4) smoothed with a 6 $\mathrm{mm}$ Gaussian kernel. Subsequently, the fMRI images were co-registered with their anatomical counterpart using Anima and then linearly transformed to the Montreal Neurological Institute (MNI) template space. The residual physiological noise was then reduced using CompCor as implemented in Nilearn ${ }^{2}$ The diffusion images were preprocessed using the following pipeline [10]: the volumes were (1) corrected for eddy image distortion, (2) rigidly realigned to remove subject motion, (3) denoised using blockwise non-local means filtering, (4) skull stripped using an atlas-registration-based method, (5) co-registered with their anatomical counterpart and then linearly transformed to the MNI template space. Whole brain deterministic tractography was then performed using fiber assignment by continuous tracking method ${ }^{3}$. Finally, the preprocessed images were partitioned into $N=360$ regions of interest (ROIs) using the Glasser atlas [11]. The structural connectivity (SC) measure represents the number of streamlines connecting each pair of ROIs divided by the sum of their volumes and stored in the adjacency matrix, called A. Then, the degree matrix $\mathbf{D}$ was computed, containing on its diagonal the degree of the corresponding node (sum of all the weighted links established by a regional node with all the others). The functional connectivity (FC) matrix was obtained thus by extracting the average time-series from each ROI and by calculating the correlation using Pearson's correlation coefficient.

\subsection{Graph Signal Processing}

In this paper, we are interested in the analysis of a functional signal $\mathbf{x} \in \mathbb{R}^{N}$ on a structural graph. We defined a graph $\mathcal{G}=\{\mathcal{V}, \mathcal{E}, \mathbf{A}\}$, where $\mathcal{V}$ is a set of nodes, $\mathcal{E}$ is the set of undirected edges in $\mathcal{G}$ and $\mathbf{A} \in \mathbb{R}^{N \times N}$ is the adjacency matrix previously defined, using the structural connectome. We defined a shift operator based on the definition of the symmetric normalized Laplacian $\mathbf{L}=\mathbf{D}^{-1 / 2} \cdot(\mathbf{D}-\mathbf{A}) \cdot \mathbf{D}^{-1 / 2}$, given its ability to capture and highlight patterns residing on

1 https://anima.irisa.fr

2 http://nilearn.github.io/

3 https://anima.readthedocs.io/en/latest/ diffusion.html\#deterministic-tractography the graph $\mathcal{G}$ [5]. Since the graph Laplacian $\mathbf{L}$ is symmetric positive semidefinite, the graph shift operator can be factorized as $\mathbf{L}=\mathbf{U} \cdot \Sigma \cdot \mathbf{U}^{T}$, where $\Sigma=\operatorname{diag}\left[\lambda_{0}, \ldots, \lambda_{\mathrm{N}-1}\right]$ is a diagonal matrix containing the positive eigenvalues. Using the graph Fourier transform, the spectral representation of $\mathbf{x}$ defined on the graph can then be expressed as: $\hat{\mathbf{x}}=\mathbf{U}^{T} \cdot \mathbf{x}$.

In the graph setting, the eigenspectrum (collection of ordered eigenvalues) conveys a concept of frequency analogous to classical Fourier analysis [12]. Eigenvectors obtained from the singular value decomposition of $\mathbf{L}$ can be employed as graph filtering operator. To extract certain graph frequency groups, a diagonal graph filtering matrix $\mathbf{F}=\operatorname{diag}\left[f\left(\lambda_{0}\right), \ldots, f\left(\lambda_{\mathrm{N}-1}\right)\right]$ can be defined, such that its elements are the frequency response of the graph frequency linked to the eigenvalue of $\mathbf{L}$.

For fMRI time series $\mathbf{X} \in \mathbb{R}^{N \times T}$ defined on the graph, the graph filtered components are given by: $\mathbf{Y}=\mathbf{U} \cdot \mathbf{F} \cdot \mathbf{U}^{T} \cdot \mathbf{X}[7]$. In the presented work, a high-pass filtering was performed in order to evaluate the contributions of the highest part of the eigenspectrum. While lower graph frequencies span over ample spatial regions, the highest part of the graph frequency spectrum denotes a greater variability in the graph signal between structurally connected nodes. To select the interval containing the $k$ highest frequencies, the graph filtering matrix $\mathbf{F}$ was then defined by: $f\left(\lambda_{\mathrm{i}}\right)=1, \forall i \geq N-k$ and 0 otherwise. In this study, the energy of the graph filtered component $\mathbf{Y}$, averaged across time, was evaluated for the $n$ th node, by $\mathcal{E}_{\mathbf{Y}_{\mathbf{k}}}(n)=\frac{1}{T} \sum_{t=1}^{T} Y_{k}(n, t)^{2}$, where $Y_{n, t}$ is the $(n, t)$-th element of $\mathbf{Y}$.

\subsection{Classification}

In this paper, we performed a supervised classification analysis that compared several connectivity features using or not GSP to differentiate patients suffering from anxiety and control subjects. First, using only functional connectivity, we compared the classification accuracy using FC matrix, the standard deviation (SD) of the rs-fMRI time series and network complex measures such as node strenght (NS), clustering (CL) and eigenvector centrality (EC). Then, the same features (SD, NS, CL and EC) using the times series projected on the graph Fourier transform (GFT) of the structural graph were computed as described in previous section and in [8]: GFT-SD, GFT-NS, GFT-CL and GFT-EC. Contrary to the method proposed in [8], the graph was build by averaging the participants' own structural matrices instead of the average human connectome project structural model. Afterwards, as described in previous section, we additionally computed the method proposed in this paper based on high graph frequency energies $\mathcal{E}_{\mathbf{Y}} \in \mathbb{R}^{N \times N}$ of graph filtered components of rs-fMRI time series. We also performed an analysis of the classification accuracy with respect to the number of frequencies selected in the high-pass graph filtering, starting from the highest graph frequency and incorporating 
descending frequencies, until considering all the eigenspectrum ( $N$ graph frequency modes). Selecting the highest part of the eigenspectrum proved to offer the best classification performance in the control-anxiety discrimination task. All the classification tasks were performed using the supervised binary Linear Support Vector Classifier (SVC) subdued to $l_{2}$ penalization and regularization parameter $C=0.01$, trained with $80 \%$ of the samples, tested with the remaining $20 \%$ and cross-validated using a stratified shuffle over 50 splits. The classification performed on these features relied on ANOVA to select the best 10 on each step, and the classification was iterated until all features $(\mathrm{N})$ were considered. The classification performance was evaluated using different metrics, such as accuracy (Acc), sensitivity (Se) and specificity (Spe).

\section{RESULTS}

The proposed method was compared with other approaches using only functional connectivity metrics and the same functional features after projection of the time series on the structural graph. Table 1 collects classification scores for such features. The best performance was obtained for $\mathcal{E}_{\mathbf{Y}}(k=96)$ (Acc $76.2 \%$, Se $73.0 \%$ and Spe $80.7 \%$ ), followed by GFT-EC (Acc $67.9 \%$, Se $67.0 \%$, Spe $68.8 \%$ ), as opposed to its unimodal counterpart EC (Acc 50.8\%, Se 53.5\%, Spe 51.2\%). The classification based on the GSP features using a combination of functional and structural information yielded overall higher performance than other approaches. These results are an indication that considering information from both functional and structural organization could improve the discrimination capability of the classifier.

Figure 1 depicts 360 classification scores of the proposed method based on energy estimation, as a function of number of frequencies selected in the high-pass graph filtering. The best classification accuracy was obtained for $k=96$ frequencies. In Figure 2 average normalized energies $\mathcal{E}_{\mathbf{Y}}(k=96)$ across time were drawn for the 360 ROIs in the two groups. Differences can be observed in the profiles of high-frequency energy distribution between control and anxiety groups. As described in [6], the brain activity patterns with high frequencies components correspond to cerebral activity decoupled with respect to structure, moreover these components can be traced back to finer localized brain spatial activations [5]. In this particular analysis, those regions corresponds to subcortical systems and more particularly to emotion networks, whose dysfunction could be related to anxiety. The 20 most relevant features were mapped in a graphical brain representation [13], as shown in Figure 3 Among them, it was found a proximity with regions in the cingulo-opercular network (lateral anterior Insula, left medial anterior Insula, left anterior Insula) and the subcortical region of the Amigdala (left Amigdala parahippocampal), both structures are known to be involved in cognitive responses and emotions processing related to anxiety [14]. The fact that the most relevant

\begin{tabular}{|c|c|c|c|}
\hline & Acc (\%) & Se (\%) & Spe (\%) \\
\hline SD & $48.0 \pm 2.6$ & $50.0 \pm 3.9$ & $48.4 \pm 3.3$ \\
\hline NS & $47.7 \pm 1.7$ & $43.0 \pm 3.5$ & $56.4 \pm 3.1$ \\
\hline CL & $53.1 \pm 2.1$ & $51.5 \pm 2.8$ & $54.4 \pm 2.8$ \\
\hline EC & $50.8 \pm 2.1$ & $53.5 \pm 3.6$ & $51.2 \pm 2.9$ \\
\hline GFT-SD & $66.2 \pm 2.0$ & $68.0 \pm 3.0$ & $64.8 \pm 3.0$ \\
\hline GFT-NS & $63.3 \pm 2.6$ & $67.0 \pm 3.6$ & $60.7 \pm 3.8$ \\
\hline GFT-CL & $57.3 \pm 2.4$ & $54.0 \pm 3.5$ & $60.0 \pm 3.2$ \\
\hline GFT-EC & $67.9 \pm 1.9$ & $67.0 \pm 3.9$ & $68.8 \pm 2.9$ \\
\hline $\mathcal{E}_{\mathbf{Y}}(k=96)$ & $\mathbf{7 6 . 2} \pm \mathbf{1 . 5}$ & $73.0 \pm 2.3$ & $80.7 \pm 1.9$ \\
\hline
\end{tabular}

Table 1. Comparison of classification scores (accuracy (Acc), sensitivity (Se), specificity (Spe)) of the features defined in Section 2.4

classification features, which were associated to nodes that behave differently from the control condition, were close to known anxiety networks, suggests that the classification selected regions that actually play a role into the expression of anxiety.

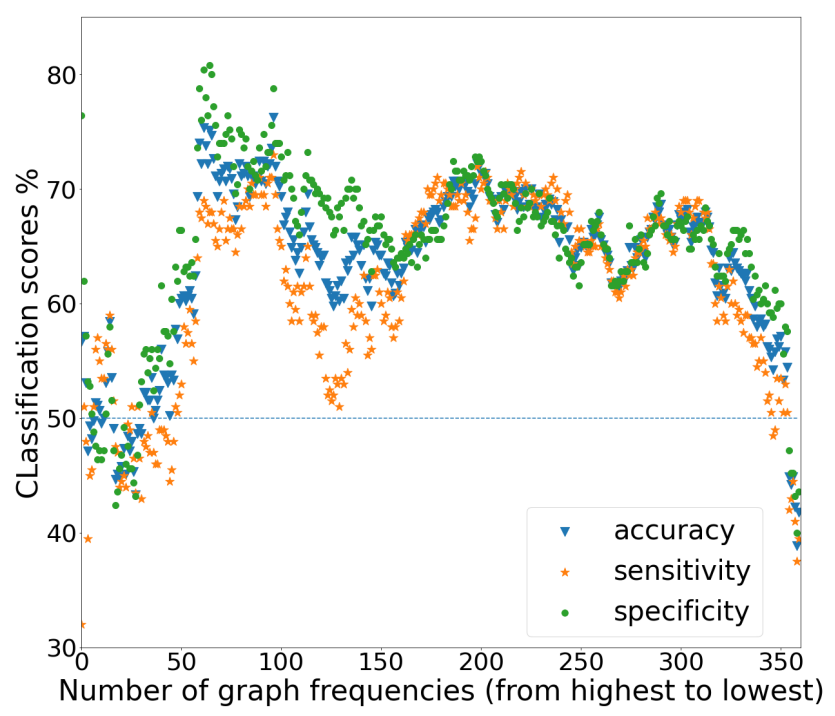

Fig. 1. Classification scores using the energy of graph filtered time series. The contributions of the elements of the eigenspectrum are considered, starting from the highest graph frequency and then incorporating the followings, decreasingly in their order.

\section{CONCLUSION}

In this work, we performed a connectivity analysis exploiting the GSP framework, to classify anxiety patients and control subjects. The proposed method was compared with approaches using only functional features and other GSP metrics. Our results demonstrated that the multimodal methods outperformed the classical unimodal graph-based approaches. 
Moreover, our results indicate that the method based on highpass graph filtering allows us to discriminate more accurately between anxiety patients and control subjects.

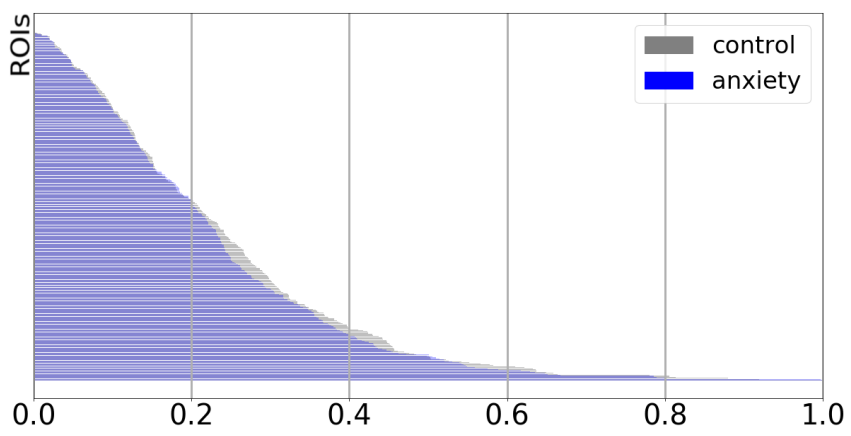

Fig. 2. Averaged energy $\mathcal{E}_{\mathrm{Y}}(k)$ across time, for each of the 360 ROIs in control and anxiety groups, in correspondence of the high frequency interval that yields the maximum value of accuracy in classification $(k=96)$.

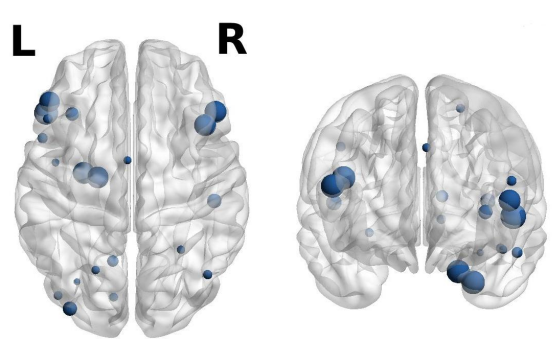

Fig. 3. Visualization of the 20 most significant ROIS in the anxiety discrimination task based on energy of graph filtered components $\mathcal{E}_{\mathrm{Y}}(k=96)$.

\section{ACKNOWLEDGMENTS}

No funding was received for conducting this study. The authors have no relevant financial or non-financial interests to disclose.

\section{COMPLIANCE WITH ETHICAL STANDARD}

This research study was conducted retrospectively using human subject data made available by Child Mind Institute Biobank, regulated by Data User Agreement. All study protocols were approved by an institutional review board provided by Advarra, and informed consent was obtained in all cases.

\section{REFERENCES}

[1] K. Holzschneider and C. Mulert, "Neuroimaging in anxiety disorders," Dialogues Clin Neurosci., vol. 13, 2011.
[2] E. Bullmore and O. Sporns, "Complex brain networks: graph theoretical analysis of structural and functional systems," Nature Reviews Neuroscience, vol. 10, 2009.

[3] F.V. Farahani, W. Karwowski, and N.R. Lighthall, "Application of graph theory for identifying connectivity patterns in human brain networks: A systematic review," Frontiers in Neuroscience, vol. 13, pp. 585, 2019.

[4] J. Zimmermann, J.D. Griffiths, and A.R. McIntosh, "Unique mapping of structural and functional connectivity on cognition," Journal of Neuroscience, vol. 38, no. 45, pp. 9658-9667, 2018.

[5] W. Huang et al., "Graph signal processing perspective on functional brain imaging," in Proceeding of IEEE. IEEE, 2018, vol. 106, pp. 868-885.

[6] M.G. Preti and D. Van De Ville, "Decoupling of brain function from structure reveals regional behavioral specialization in humans," Nat Commun, vol. 10, October 2019.

[7] S. Sihag et al., "Multimodal dynamic brain connectivity analysis based on graph signal processing for former athletes with history of multiple concussions," in IEEE Transactions on Signal and Information Processing over Networks, 2020, vol. 6, pp. 284-299.

[8] A. Brahim and N. Farrugia, "Graph fourier transform of fmri temporal signals based on an averaged structural connectome for the classification of neuroimaging," $\mathrm{Ar}$ tificial Intelligence in Medicine, vol. 106, pp. 101870, 2020.

[9] L.M. Alexander et al., "An open resource for transdiagnostic research in pediatric mental health and learning disorders," Nature - Scientific Data, vol. 4, December 2017.

[10] J. Coloigner et al., "White matter abnormalities in depression: A categorical and phenotypic diffusion mri study," Neuroimage : Clinical, vol. 22, 2019.

[11] M.F. Glasser et al., "A multi-modal parcellation of human cerebral cortex," Nature, vol. 536, pp. 171-178, July 2016.

[12] D.I. Shuman et al., "The emerging field of signal processing on graphs- extending high-dimensional data analysis to networks and other irregular domains," IEEE Signal Processing Magazine, vol. 30, 2012.

[13] M. Xia, J. Wang, and Y. He, "Brainnet viewer: a network visualization tool for human brain connectomics," PLoS One, vol. 8, no. 7, 2013.

[14] C.M. Sylvester et al., "Functional network dysfunction in anxiety and anxiety disorders," Trends Neurosci., vol. 35, no. 9, pp. 527-535, June 2012. 